\title{
EDITORLAL
}

\section{RESEARCH, MANUSCRIPTS AND PUBLICATIONS ...}

One of the indicators of research productivity of scientists is the output of research papers. Quality research papers can only be published based on significant findings of research studies. The question is: do we publish enough to reflect the correct level of productivity? Could we improve on this aspect whenever important results are available?

Today only a handful of scientists in our country, conduct productive research. However, some of them even have excuses for not publishing their findings on time. The low level of research productivity does not necessarily mean lack of good material to publish. This is partly due to our own research culture. Is there a way to break this mould that we have trapped ourselves in? There are several aspects worth looking into. First, one has to face the challenge of "finding time" to write. Then, one must move out of the shell of "full paper or nothing" misconception. The moment significant results are obtained, quick action is a must. The result could be a full paper, a short communication, a correspondence, or even a letter. The important thing is to document the findings as soon as possible. Of course, not all scientists are blessed with the ability to write well. However, they (especially the younger generation) must not make it an excuse or consider as an obstacle for writing up a paper, since there are many ways in which this could be overcome.

Writing alone is not sufficient. The manuscript has to be of an acceptable standard for publication. Scientists living in developed countries (including our own expatriates), naturally become part of the research culture in that environment. Driven by necessity, they publish their research findings on time. Almost all of the journals in which they publish are "international". Unfortunately, this is not the case with the local scientists. Times have changed and today, anybody should be able to find useful hints on the internet on "how to write a publishable paper". In this respect, it is worth highlighting a few common points not usually found on these sites. For example, if a revision of a paper leads to rerevision, then it is usually the fault of the author/s. Some authors simply do not address all points raised by referees. It may be that, some purposely avoid a few referral points thinking they will go unnoticed. Timely returning of the manuscript with all necessary corrections, and any clarifications particularly when the author is not in agreement with the referees, would avoid any delays or rejection.

The thought that some form of advanced statistical analysis is always needed is another myth. What is necessary is an appropriate and valid analysis. A simple table of means and standard errors, perhaps with some tested correlations, if properly computed, would be more appropriate than ANOVA with highly unequal group sizes without checking for underlying assumptions! Pre-mature conclusions and generalization of findings beyond the scope of the experiment, is another problem that should be avoided. A typical example is the study of growth curves over a limited time range. In most cases, the response would be linear whereas actual growth pattern if studied over the entire range, would certainly be non-linear. Finally, it is worthwhile to realize that one could improve a manuscript for publishing on time by simply paying careful attention to minute details. 